FEDSM2009-78014

\title{
DRAFT \\ TEACHING HOW TO USE THE CFD APPROACH BY AN EXAMPLE: HYDRODYNAMICS WITHIN A PASSENGER CAR COMPARTMENT IN MOTION
}

\author{
Geanette Polanco \\ Simón Bolívar University \\ Caracas, Dtto. Fed., Venezuela
}

\author{
Nelson García \\ Simón Bolívar University \\ Caracas, Dtto. Fed., Venezuela
}

\author{
Luis Rojas \\ Simón Bolívar University \\ Caracas, Dtto. Fed., Venezuela
}

\begin{abstract}
The CDF methodology is applied to the study of the air flow around a 2-D car and its interaction with the cabin internal air. The flow visualization or computational works enable engineers to calculate different car characteristics like drag coefficient, external and internal air flow patterns, etc. Therefore, the teaching of this approach to student is a very important task to take into account in the formation process of new engineers. This work shows the numerical simulation of a specific passenger car compartment configuration solving the Navier-Stokes equations along with the k-e turbulence model using the finite volume method. The indoor air flow is produced by the interaction between the cabin inner air with the external flow through two glass windows (one in the front seat and one in the back seat). This configuration represents a common situation for the passenger car compartment. The study covers two different car speeds, 50 and $100 \mathrm{~km} / \mathrm{h}$. The flow field is studied in both steady state and transient conditions with time step of $0.01 \mathrm{~s}$, for both car speeds, $50 \mathrm{~km} / \mathrm{h}$ and $100 \mathrm{~km} / \mathrm{h}$. The different steps of the CFD work are commented to show to the reader the distinct states that must be cover in this kind of work. As results of the detailed methodology followed, the influence of the domain size on the flow fields is highlighted, the requirement of a better mesh quality is exposed and flow field results are analyzed using two different forms of graphic representations. The results show the physics behavior of the flow and the presence of flow structures, as for instance, indoor air recirculation zones delimited by internal seats, as well as, the vortex presence at the back of the cabin.
\end{abstract}

Keywords: CFD teaching, Flow visualization, recirculation zones.

\section{INTRODUCTION}

The constant increases of costs of doing experimental tests for many industrial applications impulses the developed of computational means or CFD to characterize new designs. The computational tools solve the governing flow equations, Navier Stokes equation and continuity, throughout a computational domain, which represents the geometric characteristics of the physical space of the problem studied, that allows obtaining the flow fields relevant to the phenomenon, as for instance velocity, pressure, temperature, turbulence or electricity fields. Therefore, it is possible to achieve the characterization of the different designs in relatively low cost and time. The output of the computational work is the visualization of the whole phenomenon or part of it, based on the premises and specific objectives of the study.

The aim of this work is to use the study the air flow within a passenger car compartment when it is in motion with two of the glass windows opened as an example of the application of a CFD tool. The model selected is a simplified version of the cabin with two open windows, which is built based on prismatic shapes that are usually of the most interest in others areas of applications, such as, flow around buildings, towers and bridge decks. This approach helps to extend the understanding of the mechanism of wake formation in relation to the object shape and flow conditions. To obtain useful results for the simulation of flow, it is necessary to have a sufficiently fine computational mesh with a correspondingly large number of mesh cells in the flow domain and a domain sufficiently large to cover the all phenomenon to be studied. These two considerations have major implications on computational resources, both memory and CPU time, required for the simulation, especially for the flow computation and solution analysis. As well as, the correct selection of the turbulence model to be used along with the Navier Stokes equation and continuity. According with 
objectives and aims of this work the standard k- $\varepsilon$ turbulence model is selected, instead of LES turbulence model, which has been used recently in this kind of flow simulation, $[1,2]$.

This work shows in an ordered and analogical way the procedure to be followed to apply a CFD technique to any problem. However, it is not the intention to produce a guide that discharges books whit the fluid dynamic concepts.

\section{MODELING PROCESS}

The most important part of the modeling process is the actual definition of the objectives and aims of the study to be performed. Frequently, the user needs to define the particular aspects to be considered and the aspects to be neglected in the resolutions of the governing equations, in order to achieve a realistic representation of the problem. In the case of this work the main objective is the study of the interaction of the external flow of the cabin and the internal part of the cabin. Although the whole car fuselage can introduce perturbations in the velocity field around the cabin, it is assumed that at the windows high the flow is not affected by those perturbations. Therefore a 2D simulation can be used instead a full 3D simulation. Additionally, it is very important to establish if the phenomenon studied have a steady state behavior or a transient behavior, moreover, it is necessary to answer the question $i$ do the objectives and aims of the study require to simulate a transient behavior?.

Once the physics aspect is clearly defined, the next step is the building of the representation of the problem to be studied. Here, it is useful to transmit to the student that the best option to do CFD is not necessarily the option with the most complicated and exact geometry. Even, using CFD techniques the simulation of the complete car geometry results very high resources consuming. The use of a simplified version of the actual geometry instead the full scaled geometry is a common practice in the resolution of real problems [3, 4]; however, it is essential to be aware of the implications of each simplification can have on the overall behavior of the results seeking in the simulation.

The geometry used as computational model in this work contains the cabin space, without any other component of the fuselage, which represents a simplify version of the passenger car compartment, as it can be seen in Figure 1. The passenger car compartment is drawn as a plane extruded in high, with walls of $5 \mathrm{~cm}$ of thickness and the two internal obstacles that represent the back of seats inside. The computational domain considers a space around the passenger car compartment geometry required take into account the interaction of the internal and external flow fields. It is recommendable to express the domain size based on characteristics lengths rather that the dimension itself. In this case the length $\mathrm{L}$ and wide $\mathrm{W}$ of the model were used as such, as represented in table 1. There is not rule to establish the size of the domain to be modeled, in fact that is a variable to consider in the analysis of the results. Mainly, the target in this point is to define a domain with sufficient space to enclose the phenomenon studied avoiding any interference with the borders of the domain. Table 1 shows two domain sizes selected to do the simulation of the case proposed.

Table 1. Relevant dimensions of the computational model

\begin{tabular}{|l|c|c|}
\hline \multicolumn{1}{|c|}{ Dimension } & $\begin{array}{c}\text { Initial } \\
\text { domain }\end{array}$ & $\begin{array}{c}\text { Second } \\
\text { domain }\end{array}$ \\
\hline Cabin length, L, (m) & 2 & 2 \\
\hline Cabin wide, W, (m) & 1.3 & 1.3 \\
\hline $\begin{array}{l}\text { Distance proportion at the } \\
\text { front of the cabin }\end{array}$ & $1 \mathrm{~W}$ & $1 \mathrm{~W}$ \\
\hline $\begin{array}{l}\text { Distance proportion at the } \\
\text { back of the cabin }\end{array}$ & $2 \mathrm{~L}$ & $3 \mathrm{~L}$ \\
\hline $\begin{array}{l}\text { Distance proportion close } \\
\text { to the cabin (Each side) }\end{array}$ & $1.5 \mathrm{~W}$ & $1 \mathrm{~W}$ \\
\hline
\end{tabular}

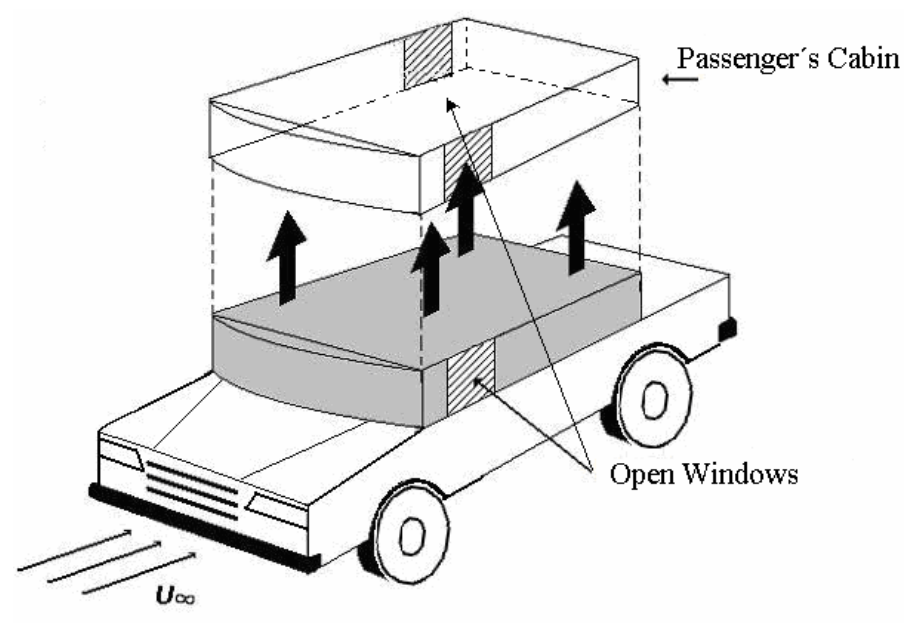

Figure 1. Schematic representation of the car compartment

The real flow conditions must be also represented in the simulations, to do that some boundary conditions must be imposed at the borders of the domain. The boundary conditions used to reproduce the actual conditions at the passenger car compartment movement are constant pressure for the laterals borders and the back of the domain, constant velocity at the front of the domain (50 y $100 \mathrm{Km} / \mathrm{h}$, respectively) and symmetry condition at the top and bottom parts of the domain. The selection of these conditions is based on the fact that the domain is sufficient to achieve a complete developed flow condition at the back of the cabin and to have a parallel flow at the sides of the cabin.

To solve the governing equations trough the domain, it is necessary to create a spatial mesh. The criterion to create the mesh varies from geometry to geometry. As presented in table 1 two different domain size were simulated. Both with variable size mesh built to cover the internal flow details and the external flow patterns using a structured mesh. The mesh is selected to be denser close to the outside border of the car compartment as well as the internal mesh (see figures 2 and 5). The density of the mesh can vary according to the area inside the domain and the type of flow that is expected in that area. 
Figure 2 shows the domain and the mesh used at first to do the simulations.
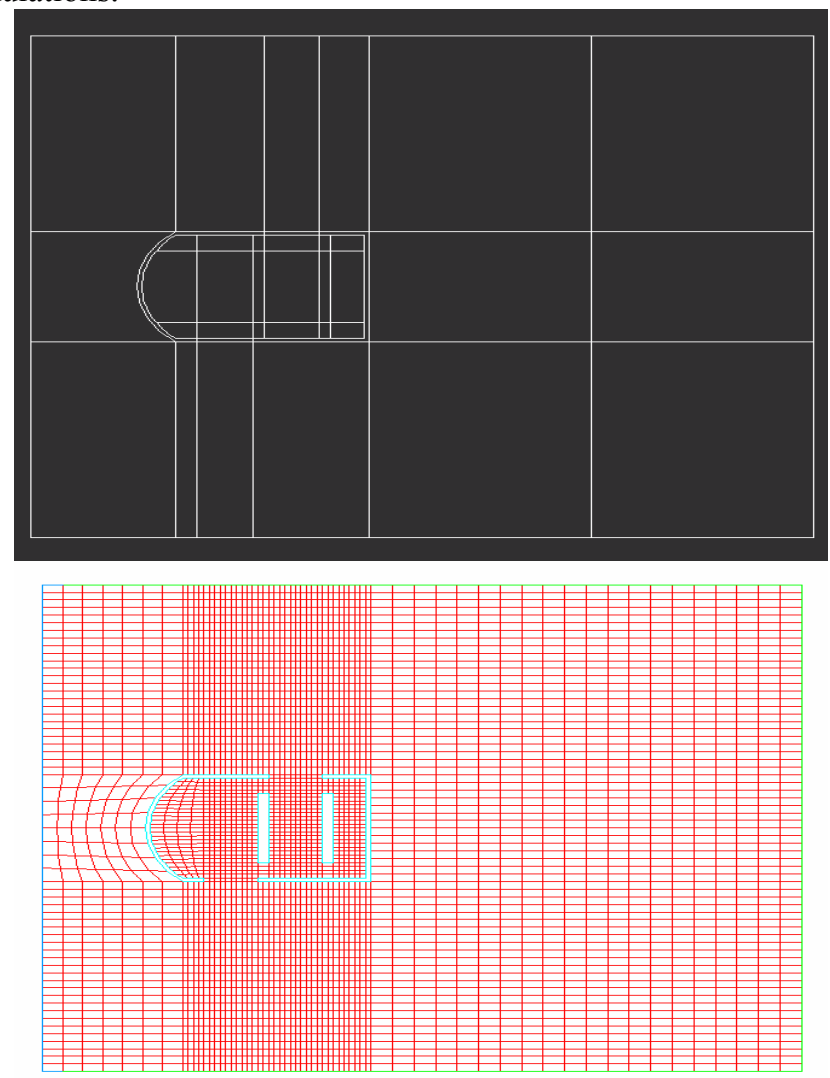

Figure 2. First domain tested. A) Upside view of the geometry modeled. b) Upside view of the mesh used and the boundary conditions proposed

At last but not less important is how to know when the simulation has obtained the results. Well, the answer of this question has two main roots. The first root is related with the criteria of tolerance imposed to the solution to stop. These criteria basically established how much difference or error is required between iteration and the next. Ideally, this difference must tend to cero. However, in practice these values are set to numbers distinct of cero but relatively smaller. The second root is related with the kind of precision and the numerical schemes set at the beginning of the simulation. Double or single precision implies more or less requirements to the simulation to stop, as well as, the usage of high order numerical schemes effectively require to achieve low level of errors before the simulation stops.

\section{DOMAIN AND MESH CHECKING PROCESS}

As it has been introduced in previous sections, there are some aspects that need to be checked before simulations results can be taken as definitive. This checking process is made doing the actual simulations and analyzing the results. The checking of the boundary conditions effects on domain, as well as, the checking of the influence of the mesh quality on the obtained results is presented as follow. The procedure to do it is based on the analysis of the behavior of the results in the zones close to the borders where the boundary conditions where applied. If the domain is not sufficient then the boundary condition can not be fulfilled. If the quality of the mesh is not appropriate then flow fields can not observer in details. For instance, Figure 3 shows that the length of the cabin at the back of it is insufficient to achieve the complete development of the expected vortex structure behind the passenger car compartment. This expectation is based on a previous knowledge of flow around solid. Additionally, it is necessary to keep in mind that the constant pressure boundary is related with no presence of any gradient of velocity in the perpendicular direction of the boundary. Condition did not achieve in this case (see Figure 3). Actually there is a recirculation zone with an area of entrainment close to the domain border; therefore the imposition of a constant pressure boundary condition can not be satisfied. It situation suggests that the domain must be increased. So, an enlargement the domain is needed. The lateral and front seem to be sufficient.

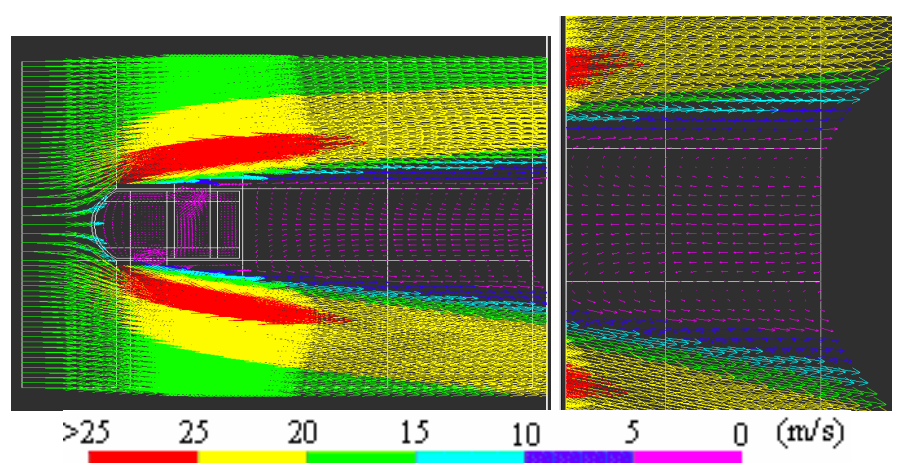

Figure 3. Velocity field in the whole domain for a car speed of $50 \mathrm{Km} / \mathrm{h}$ case in steady state condition. b) Detail of the velocity field close to the domain border at the back of the model. Vectors are presented with a scale factor of 10

The indoor part of the model shown in Figure 4 represents a good example of a poor mesh quality in some zones. Although, the flow in the cabin looks quite develop and in interaction with the outside flow. It can be observer the element in the transition zone between inside and outside of the cabin is not smooth, with means there are not sufficient elements in this area. The relative big spacing between arrows at the front of the model is a signal of a mesh refining is needed. Major resolution is also needed in the back part of the cabin to achieve a better vector definition. As a result of the previous analysis a new mesh with more elements in front of the passenger car compartment, and a softer transition between size elements corresponding to indoors and outdoors positions is proposed. The new domain and mesh shown in Figure 5 also need to be tested, in order to assure that the criteria of the complete developed flow are achieved. If not, another domain and mesh need to be proposed and tested. This iterative procedure will be 
repeated until the independence of the results is proved by both the domain size and the mesh quality.

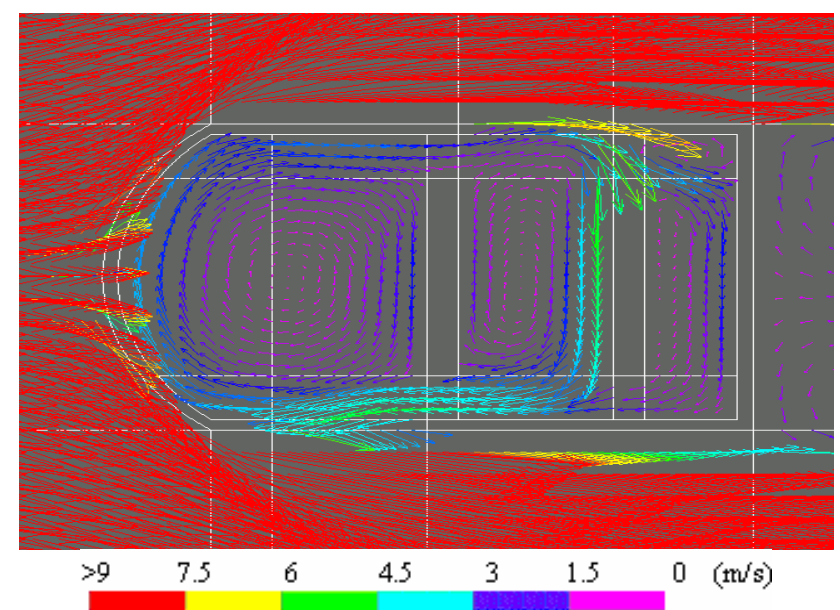

Figure 4. Details of the velocity fields indoor flow corresponding to a car speed of $50 \mathrm{~km} / \mathrm{h}$ (left side) in transient condition

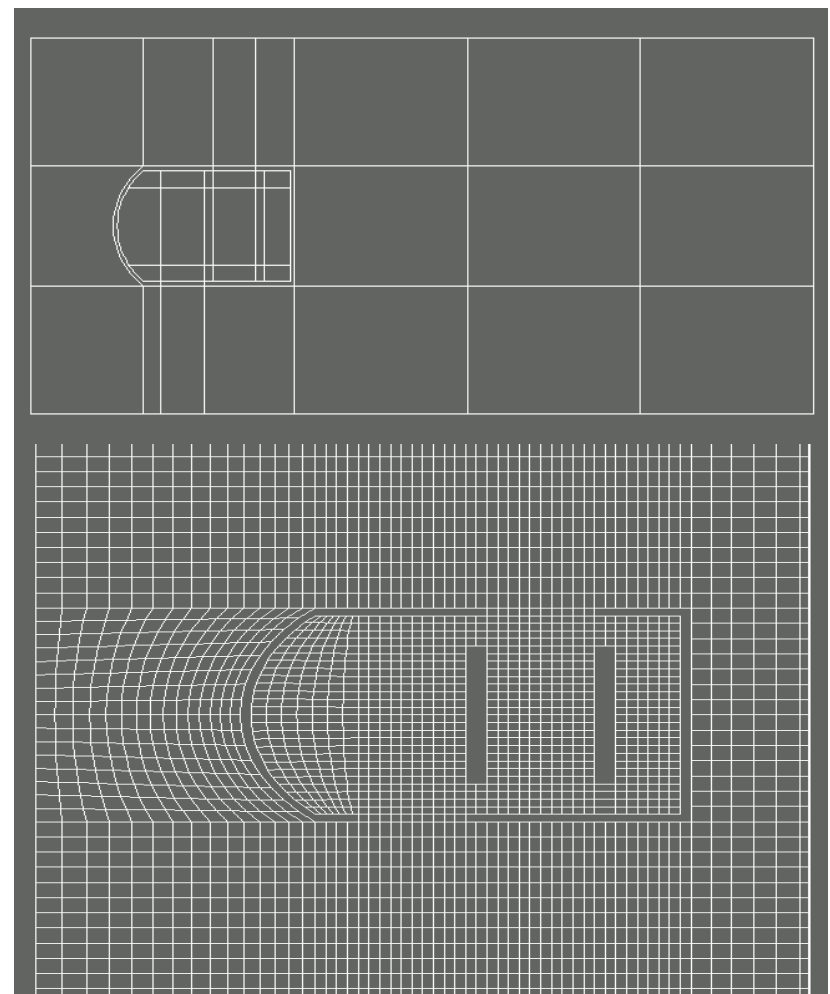

Figure 5. Upside view of the new domain modeled. Detail of mesh closer in and out of the cabin

The results of this new mesh can be observed in Figure 6 . In this case it is clear that the velocity at the rear border of the domain does not have any vortex or recirculation zone. Although, it is not perfectly a constant along the border as it should be, at least the velocity is parallel to the horizontal axis for the whole border, which means that the vortex structure behind the cabin is completely inside the domain. Also, the mesh inside the cabin gives a more detailed flow field.
Therefore considering the aims of this work this domain size and mesh distribution is considered sufficient to do the simulations.

In case that the mesh need to be more dense, the same procedure must be done again for a fixed domain size, even with a denser mesh in order to assure that the obtained result does not depend on the mesh density. The criterion of comparison can be the velocity profile at a particular region, which can be the outlet of the domain or any other location.
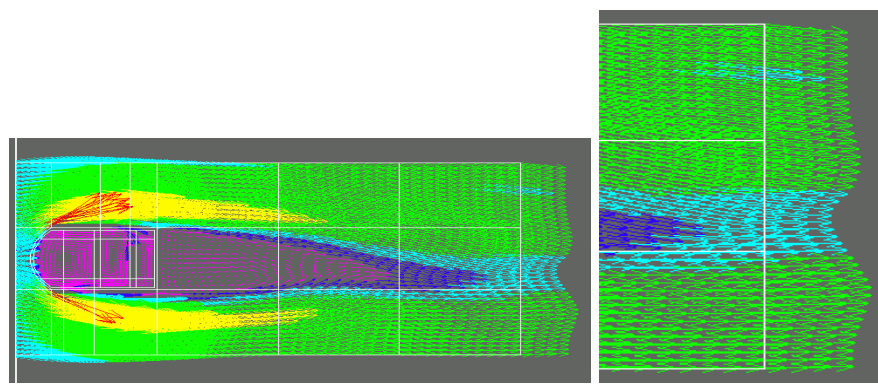

Figure 6. Velocity fields for a car speed of $50 \mathrm{~km} / \mathrm{h}$ in steady state and transient conditions (upper part). Velocity field for a car speed of $100 \mathrm{~km} / \mathrm{h}$ in transient condition (lower part)

\section{RESULTS}

In general all CFD package can delivery result in graphic and numerical (data file) forms. Here it is shown two different types of graphic representation of result, vector velocity fields and contours fields are only two of the graphic kind. The initial type corresponds to a representation of the velocity magnitude using a color scale over vector that follow the direction of the flow, whose are already scaled according to the velocity magnitude. The contour fields do not show expressively the flow direction. They show using a degradation of color map the magnitude the parameter chosen. However, using the fluid dynamics knowledge about the behavior of the flow, the interpretation of the flow direction can be extracted indirectly. This means, that the understanding and capability of analysis of results are intimately related with the application of fluid dynamics knowledge.

The results obtained from a CFD simulation will cover different variables as velocity, pressure, turbulence variables, temperature if it was considered and others. Hereafter, some of the obtained results for the studied case are presented. Velocity fields, pressure fields, kinetic energy fields and energy dissipation fields were selected for two different car speed modeled. Car speeds of $50 \mathrm{~km} / \mathrm{h}(\operatorname{Re} 200000)$ and $100 \mathrm{~km} / \mathrm{h}$ ( $\operatorname{Re} 400000)$ were simulated in transient condition. The resulted fields can be taken in any part of the domain. The location to be shown will obey to the concept that wants to be explored or explained.

Figure 7 shows the velocity field for two different velocities. This simple superposition of figures for two case highlight the versatility of the use of CFD tool to do a study instead of doing a experimental test. The only requirement to do these two cases is change the magnitude of the velocity in the 
inlet boundary. It is clear that the vortex structure behind the cabin keeps the general trend for both cases studied. That is reasonable based in the fact that the Reynolds number for both cases correspond to a turbulent condition and therefore the interaction of the mechanisms involved are similar. The vortex structures behind bodies has a transient condition itself, [2]. The domain for this case allows the flow to develop the vortex structure behind the cabin and no recirculation is presented in any boundary. The comparison between the work presented in this paper and the flow field measurement around a square cylinder by van Oudheusden, B.W. [5], is not possible. However, taken as general point that both geometries consider a rectangular based shape, the comparison suggests that the interaction with the inside of the cabin plus the modification at front of the square shape produce and enlargement of the turbulent structure behind the cabin studied, which for a $\mathrm{Re}=$ 20000 seem to be of one time the size of the square tested.
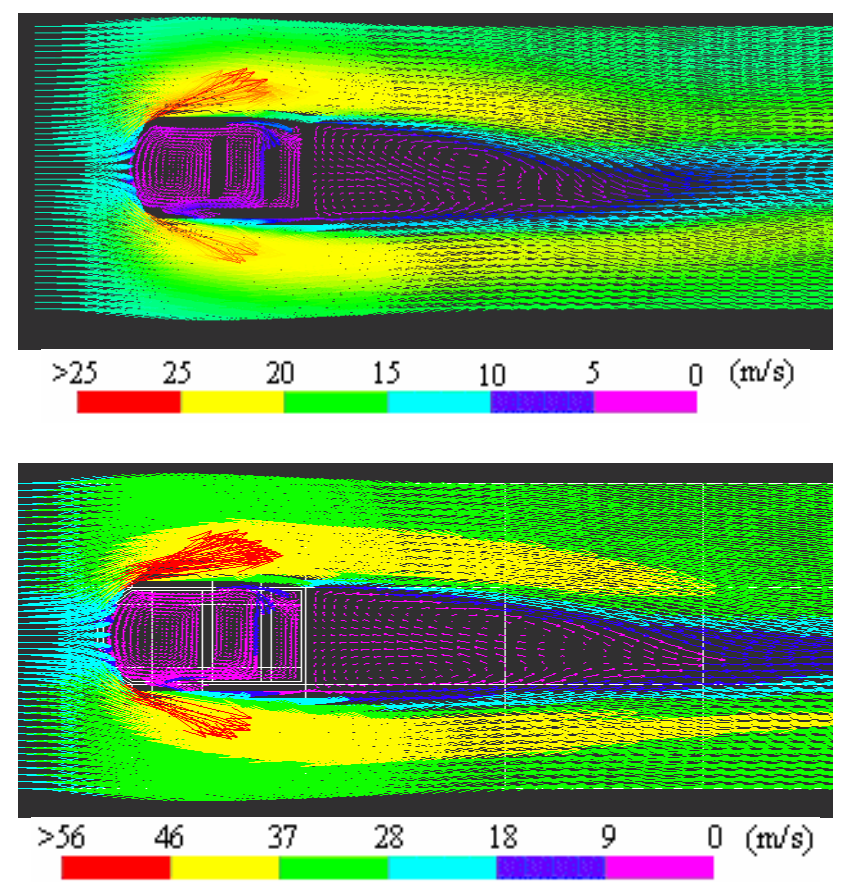

Figure 7. Velocity field for a car speed of $50 \mathrm{~km} / \mathrm{h}$ transient conditions (upper part). Velocity field for a car speed of $100 \mathrm{~km} / \mathrm{h}$ in transient condition (lower part). Vectors are presented with a scale factor of 10

There is a internal flow recirculation and the interaction at the glass windows location within external and internal flow. The trend of the internal flow suggests that the main direction of the flow is from the back window to the pilot window. The main trend is in agreement with the presence of a lower pressure at front part of the cabin due to the closeness of the stagnation point to the pilot window, as well as, the pressure recovery along the axis flow, which produces a larger pressure at the back rear window location.
Figures 8 and 9 show the pressure fields for the same two cases of $50 \mathrm{Km} / \mathrm{h}$ and $100 \mathrm{~km} / \mathrm{h}$ mentioned in Figure 7. This figure presents the whole variation of the pressure along the whole domain. The pressure fields are directly related with the velocity profiles presented in the domain. For instance, the region located in front of the cabin of corresponds to the highest pressure and the lower velocity magnitude created by the deviation of the flow due to the present of the cabin in the way of the flow, named as stagnation point. Based on the degree of detail that is needed to do the study or the range of values obtained in the simulations it is always possible to adjust the sensibility of the legend used in the map.

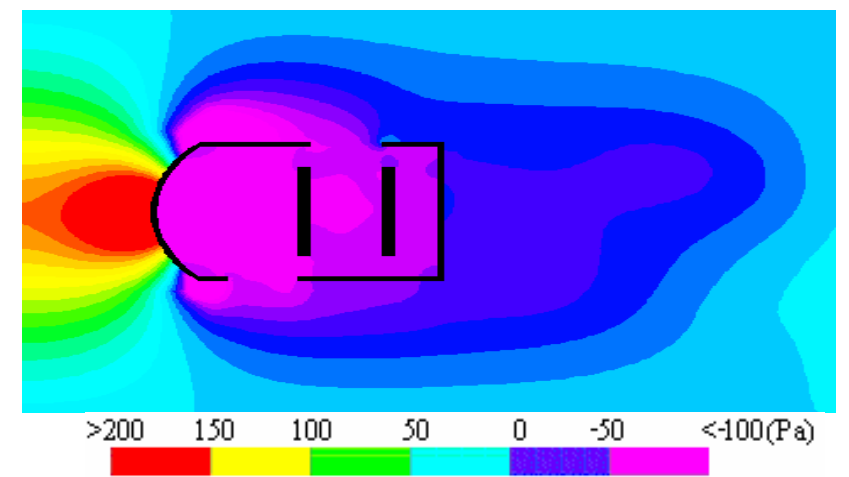

Figure 8. Pressure field for a car speed of $50 \mathrm{~km} / \mathrm{h}$ in transient condition

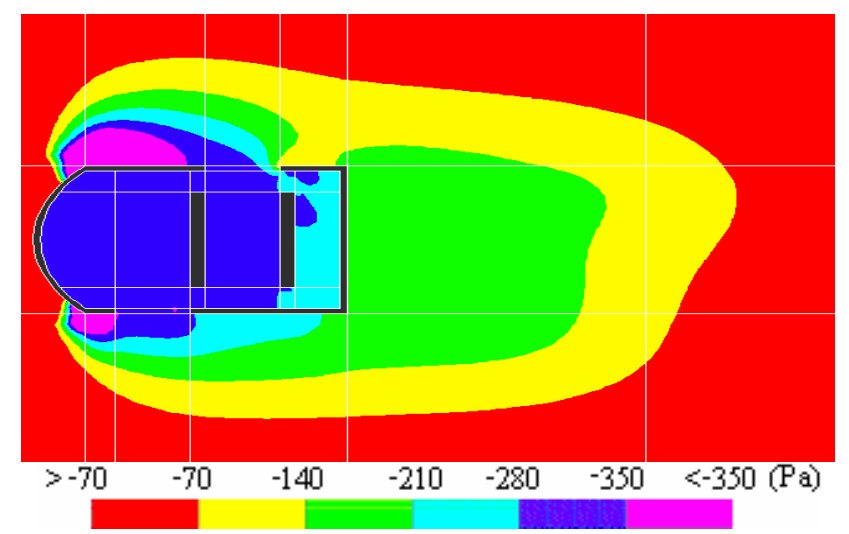

Figure 9. Pressure field for a car speed of $100 \mathrm{~km} / \mathrm{h}$ in transient condition

The distribution of the kinetic energy of the flow, shown in Figure 10, is other very important parameter to characterize. This parameter indicates the zones were the effects of the fluctuating components become important, indicating the areas with high level of turbulence in the domain. For this case the instabilities are located basically at the back of the cabin inside the vortex structure. This situation corresponds with the expected flow pattern around a solid; however it is clear that there is a connection between the vortex structure and the areas around the open windows. This connection shows the exchange the momentum between the external and internal flow. This 
exchanging increases with the speed of the car, as well as, the size of the vortex structure behind the car. The shape of the distribution of the kinetic energy seem to be in agreement with the $u$ fluctuating component of the velocity field of the flow around a square cylinder presented by van Oudheusden, B.W. [5] for a case corresponding to a $\mathrm{Re}=10000$.
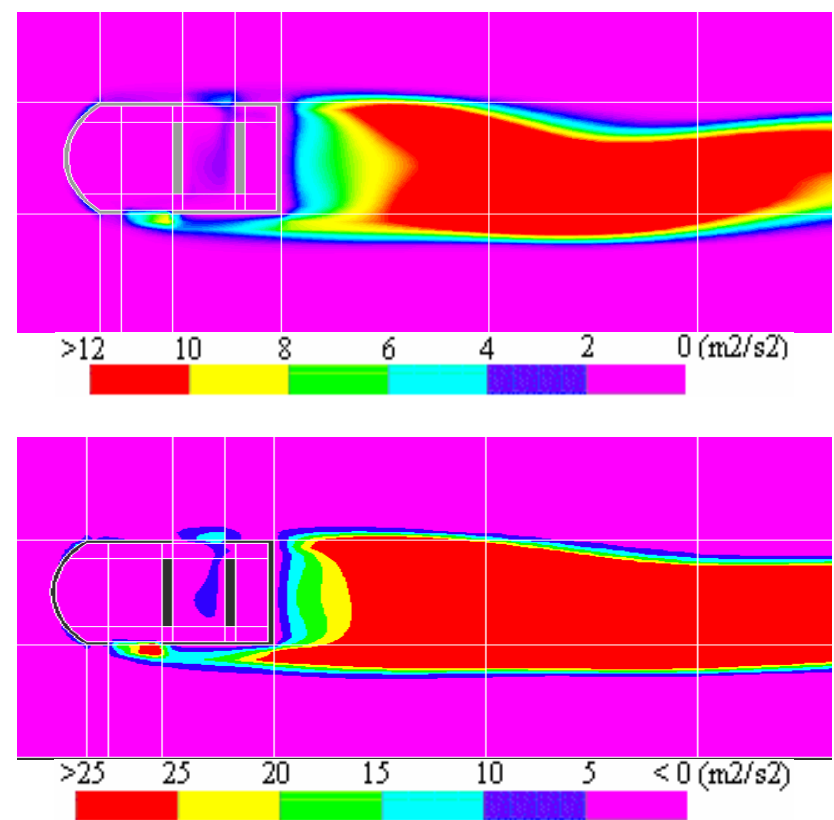

Figure 10. Specific mean turbulent kinetic energy field for a car speed of $50 \mathrm{~km} / \mathrm{h}$ (upper part) and to a car speed of $100 \mathrm{~km} / \mathrm{h}$ (lower part), both in transient conditions

The dissipation of turbulent energy shown in Figure 11 is also an indicative of the type of phenomenon is studied. Beside the scale of this two figures are not the same, it is clear that the behavior is similar. It is possible to observe that the zones with more energy dissipation are located to the outside part of the turbulent structure behind the cabin. This situation is related with the interaction with the main flow. Meanwhile the maximum kinetic energy is located at the centre of the vortex structure behind the cabin. This zone is characterized for the area with major velocity gradient, therefore more energy is dissipated. A new zone with important values of those parameters is observed close to the pilot glass.
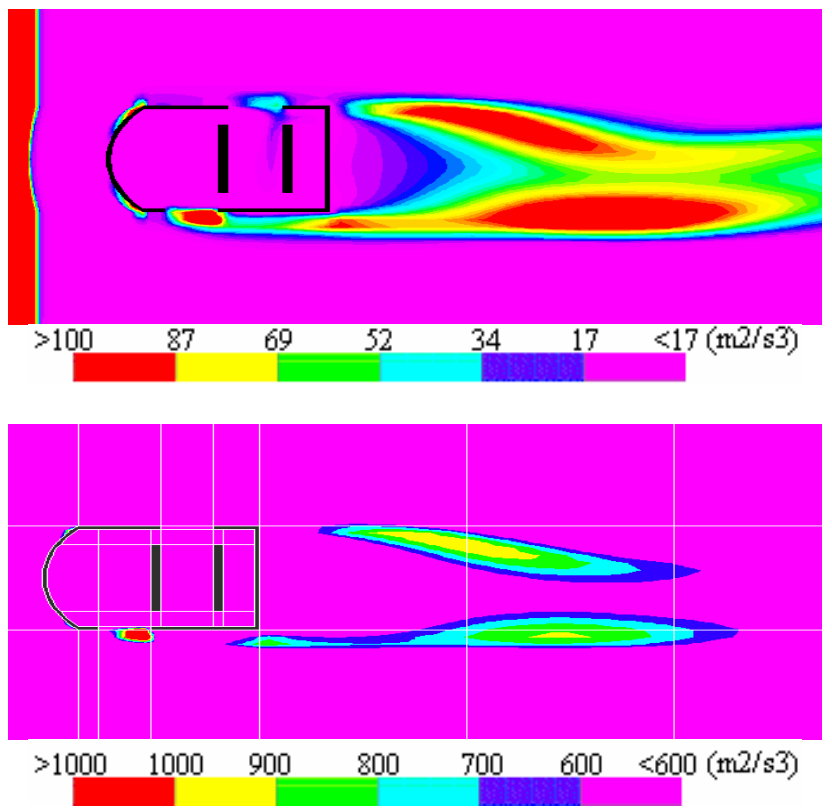

Figure 11. Specific turbulent kinetic energy dissipation field for a car speed of $50 \mathrm{~km} / \mathrm{h}$ (upper part) and to a car speed of $100 \mathrm{~km} / \mathrm{h}$ (lower part), both in transient conditions

\section{CONCLUSIONS}

The use of CFD technique allows the student to apply the basic concepts of fluid dynamics in the study and analysis of a new designs or prototypes in any area of engineering. CFD is a computational tool and therefore it can not overcome in any situation the understanding of the physics involves in the problem studied by the user. The success of the CFD application to a particular problem is based on the correct representation of the reality in every single phase of the modeling process and the correct interpretations of the obtained results.

\section{ACKNOWLEDGMENTS}

To the Fluid Mechanics Laboratory of the Simón Bolívar University for the use of the space and the resources.

\section{REFERENCES}

1. S. Krajnovic, L.D. Large eddy simulation of the flow around a simplified car model. in SAE 2004 World Congress, SAE Paper 2004-01-0227. 2004. Detroit, Michigan, USA.

2. R. McCallen, F.B., J. Ross (Eds.), Lecture Notes in Applied and Computational Mechanics / The Aerodynamics of Heavy Vehicles: Trucks, Buses, and Trains. 2004: Springer Verlag.

3. H. Lienhart, C.S., S. Becker. Flow and Turbulence Structures in the Wake of a Simplified Car Model (Ahmed Model). in DGLR Fach Symp. der AG STAB. 2000. Stuttgart University. 
4. H. Lienhart, S.B., Flow and Turbulence Structure in the Wake of a Simplified Car Model, in SAE 2003 World Congress. 2003: Detroit, Michigan, USA.

5. van Oudheusden, B.W., et al., Quantitative visualization of the flow around a square-section cylinder at incidence. Fifth International Colloquium on Bluff Body Aerodynamics \& Applications (BBAA V) (pp. 189-192). Ottawa, Ontario, Canada. 